Research Article

\title{
Promotion of VR Technology in Taijiquan Multimedia Edge Computing Technology
}

\author{
Jie Xiao ${ }^{1}$ and Mingang Guo $\mathbb{D}^{2}$ \\ ${ }^{1}$ Department of Physical Education, Zhongnan University of Economics and Law, Wuhan 430073, Hubei, China \\ ${ }^{2}$ Department of Physical Education, Wuhan University of Technology, Wuhan 430076, Hubei, China \\ Correspondence should be addressed to Mingang Guo; gmg@whut.edu.cn
}

Received 27 August 2021; Revised 8 October 2021; Accepted 16 October 2021; Published 9 November 2021

Academic Editor: Sang-Bing Tsai

Copyright (c) 2021 Jie Xiao and Mingang Guo. This is an open access article distributed under the Creative Commons Attribution License, which permits unrestricted use, distribution, and reproduction in any medium, provided the original work is properly cited.

\begin{abstract}
Taijiquan is a representative sport in my country's traditional sports. It occupies an important position in China's traditional sports. It has been rated as my country's intangible cultural heritage. Widespread and promotion of Taijiquan can promote my country's traditional culture. Using the unique advantages of Tai Chi and better development prospects, the method and path of VR technology are combined to create a new cultural atmosphere, and the active spread and promotion of Tai Chi culture are gradually carried out. VR technology is an augmented reality technology based on sensors and computers that can provide a strong visual experience. Playing the role of multimedia edge computing technology and combining VR technology with the advantages of Tai Chi itself are beneficial to change the traditional teaching methods and the spread of the Tai Chi movement. The purpose of this article is to solve some of the problems China faces in the promotion of Taijiquan and explore the role of VR technology in the spread and promotion of Taijiquan. This article uses methods such as searching literature, questionnaires, and experimental comparisons. The students in the 24-style Taijiquan elective course are the research objects, using VR technology as the teaching method of Taijiquan, scoring teaching efficiency, learning enthusiasm, students' sensory effects, the attractiveness of Taijiquan, and statistics and analysis of relevant data. The research results show that the combination of VR technology and Taijiquan teaching can increase the visual and auditory effects of students in the learning process by $28.4 \%$, the overall improvement of students' learning enthusiasm by $25.3 \%$, and the overall increase of learning efficiency by $34.8 \%$, while greatly improving Taijiquan's performance attractive. Therefore, it can be seen that the application of VR technology to Tai Chi is feasible.
\end{abstract}

\section{Introduction}

In the 1990s, the rapid development of information technology in various fields, especially in the field of information technology, has broken through the traditional education field. With the rapid development of electronic information technology, people's pursuit of display technology is no longer a simple two-dimensional level. With the development of the times, a three-dimensional imaging technology that is infinitely close to reality has emerged. VR technology (augmented reality technology) can maximize virtual information as an interactive technology in the real world. College physical education has fully introduced multimedia teaching methods. College physical education teachers should devote themselves to exploring efficient teaching methods, actively try to introduce VR technology into college physical education, effectively promote quality education, and improve the core quality of universities. Multimedia traffic has shown an explosive growth trend, and multimedia content has become the main component of current network traffic. The gap between the rapidly increasing network traffic and the slowly upgraded underlying network hardware facilities is getting bigger. At the same time, users have higher expectations of service quality. The industry and academia have begun to rethink and explore content distribution architecture. Edge computing, as a new 
concept that has just appeared in recent years, has also become a hot spot in the academic world. There are many research results that analyze the advantages of edge computing and study its application in actual scenarios. Tai Chi, a national intangible cultural heritage, is based on the traditional Chinese Confucian and Taoist philosophy, Tai Chi, and the dialectical concept of yin and yang. The changes of the five elements, the meridian of traditional Chinese medicine, the ancient Daoyin technique, and vomiting technique form a kind of traditional Chinese boxing that is both internal and external, soft, slow, light, strong, and soft. After 1949, it was uniformly adopted by the National Sports Commission for gymnastics, performances, and sports competitions for physical fitness. After China's reform and opening up, part of its original appearance was restored; thus, it was further divided into Tai Chi for competitions, Tai Chi exercises for gymnastics, and Tai Chi push hands. There are many traditional Taijiquan schools. Common Taijiquan schools include Chen style, Yang style, Wu style, Wu style, Sun style, and He style. A hundred flowers bloom. Because Tai Chi is a type of boxing formed in modern times, with many schools and broad masses, it is a very vital group of Chinese martial arts.

Taijiquan is not only a treasure of traditional Chinese culture but also the focus and difficulty of physical education teaching in ordinary colleges and universities. Taijiquan teachers in colleges and universities are not only tired of the teaching of Taijiquan but also need to teach Taijiquan in detail [1]. However, due to the influence of teaching mode, it is difficult for teachers to guide each student seriously. At the same time, due to the influence of individual learning differences among students, the effect of traditional Tai Chi teaching is very limited, and many students can only learn more knowledge from Hulu [2]. However, the introduction of VR technology broke the traditional Taijiquan teaching model, which is an upgraded model of Taijiquan multimedia teaching, which greatly improved the teaching effect of Taijiquan. In view of this, this article analyzes the application of Taijiquan teaching in colleges and universities from the characteristics of Taijiquan and virtual reality technology. VR technology is an inevitable choice for the development of college teaching [3]. The application of VR technology in Tai Chi teaching mainly includes the following aspects. First, on the basis of the VR technology, the teaching courseware can be made into each act, let students learn in sports, so that we can overcome the ability to observe specific skills from one angle in video teaching and the inability to observe the momentary action skills actually practiced by the teacher. Second, online Taijiquan teaching resources has to be developed, and the network should be used to implement distance teaching.

In order to solve some of the problems that our country faces in the promotion of Taijiquan and explore the role of VR technology in the spread and promotion of Taijiquan, this article has consulted a lot of related materials. Among them, Chi introduced in detail the origin, development process, and current branch schools of Taijiquan, pointed out that Taijiquan is my country's intangible cultural heritage, and discussed the protection measures of my country's research status of Taijiquan [4]. Cheng pointed out in his article that Tai Chi is a sport that can strengthen the body and is well loved by people and has been recognized by international sports organizations. At the same time, he pointed out that Tai Chi has not yet become international due to the limitations of its communication methods, popularization [5]. Kurokochi pointed out that VR technology involves computer graphics, sensor technology, dynamics, optics, artificial intelligence, social psychology, and other research fields. It is a new direction for the development of multimedia and three-dimensional technology. Virtual reality technology is an immersive type based on computable information. The interactive environment is a new human-computer interaction interface [6]. Ohara pointed out in the article that VR technology can enhance people's sensory effects and give people an immersive feeling by enhancing immersion. Compared with traditional $2 \mathrm{D}$ multimedia technology, it has a huge advantage and emphasizes that VR technology is a technology with broad development prospects, cutting-edge technology [7]. Zhang et al. found through research that the combination of VR technology and Taijiquan teaching can effectively improve the efficiency and interest of students in learning Taijiquan, improve students' learning experience, increase the attractiveness of Taijiquan, and facilitate the spread and promotion of Taijiquan [8]. The Tai Chi culture in Chinese martial arts is a culture of guaranteeing the body, a healthy culture, and a difference from the Western fitness culture. Therefore, the main purpose of this research is to let the Tai Chi culture go out of the country and bring people from all over the world closer or directly and make everyone feel the unique charm of Tai Chi culture in Chinese martial arts culture and enhance the status of Chinese Tai Chi culture in the world. On the other hand, learn the advanced culture in the world, learn from each other's strengths, and further promote the excellent Chinese culture. It provides us with new ideas for the promotion of Tai Chi.

In the research on the role of VR technology in the dissemination and promotion of Taijiquan, this article summarizes and analyzes the research experience and results of a large number of predecessors. In addition, this article has made some innovations in the research content and detection methods. The specific innovations are as follows: first, using the most advanced 3D full-space stereoscopic real-time shooting technology, using multipoint high-speed cameras distributed in the space to capture the movements of Taijiquan masters and restore the authenticity of Taijiquan to the greatest extent. Second, combine VR technology with Taijiquan and make Taijiquan action teaching courseware based on VR technology, so that students can overcome the problem of observing a certain technique from one angle in video teaching and the shortcomings of being unable to understand movement skills in the teacher's actual demonstration. After the spread of the times, Tai Chi has developed many genres and several schools. Nowadays, the spread of traditional Tai Chi mainly includes Chenstyle Tai Chi, Yang-style Tai Chi, Sun-style Tai Chi, Wustyle Tai Chi, Wu-style Tai Chi, and so on. The five major 
factions are known as "five-style Taijiquan." VR multimedia technology has also been further developed, which can better combine the application of virtual and reality. VR technology can comprehensively process and manage various media information such as text, data, graphics, images, animation, sound, and so on and combine various types of information well, which is also called computer multimedia technology.

\section{The Application of VR Technology and the Spread of Tai Chi}

2.1. Application of Virtual Reality Technology and Edge Computing Technology. To sum up, virtual reality technology (virtual reality, referred to as VR) refers to the use of a computer to generate a simulation environment and through a variety of special equipment to enable users to "invest" into the environment and achieve direct natural interaction between the user and the environment technology. With the development of the times, VR technology has gradually shown its superior performance and has been continuously used in many fields. At present, it is used in education and training, military applications, industry, medicine, biology, art and entertainment, visualization, and urban simulation.

VR technology is also known as spiritual technology. From the point of view of the practicality of virtual reality technology, this technology can integrate computer technology, multimedia technology, network technology, and simulation technology. This is one of the most advanced technologies in the field of computer research. It also includes professional knowledge in the fields of mathematics, optics, and mechanics [9]. In practical applications, virtual reality technology can create a virtual environment for people in a simulation manner, and users can experience visual, auditory, and tactile functions, as well as contact with the virtual environment. The real-time three-dimensional space function of VR technology and the interactive environment between humans and computers bring a good experience and change the boredom between humans and computers. New research fields are created for the communication between humans and computers, not only to apply various intelligent engineering projects but also to provide new interfaces and visual data descriptions. At the same time, it is a new way for people to explore the macro and micro worlds, and it is also a material movement that is not suitable for direct observation [10]. With the rapid development of computer technology and other advanced technologies, this technology has received widespread attention in various fields in China, and the depth of VR technology research is also expanding. The Science and Technology Committee and the Ministry of National Defense consider VR technology as a core research project. Major research institutions and universities have gradually participated in VR research and achieved amazing results. At the same time, it is also a trusted research institution and technology for VR technology. It is mainly used for the research of dynamic data distribution technology in the VR $3 \mathrm{~d}$ database and virtual environment, especially in the government research field about the object processing characteristics in VR technology. At the same time, VR technology is used to simulate and remotely operate robots. In recent years, in many applications, there is no special emphasis on virtual environments, and VR technology has become more widespread [11].

Shopping experience in a new VR breaks physical constraints and allows consumers to penetrate into it. They feel that they can experience and touch products in shopping stores. In the past two years, the number of technicians in the fields of virtual reality equipment, content, and portable game controllers has increased. I can feel the atmosphere and applause of the scene when watching the game or participating in national events such as concerts and performances, and even the interlude will remain in my memory. The above feeling can only be realized by watching live broadcasts [12]. However, the current live TV experience cannot be compared with the VR experience [13]. The application of VR technology in medical treatment mainly has two aspects. On the other hand, since it is used in medical education and training, doctors can feel the dual experience of vision and touch during virtual surgery, thereby enhancing the experience. Customers put on VR glasses and carry the structure of new homes and buildings. They are not restricted by brochures and prescribed formats and can move freely. In this way, the views of the previous real estate industry will disappear. VR travel is a $3 \mathrm{D}$ display in the form of an interactive video of the VR head-mounted display screen, which sends a 360-degree panoramic view to the user. Virtual reality is an important guide for future travel and discussion. Users can use virtual reality to achieve the purpose of planning and presentation, making travel and planning easy. At the same time, they can explore the inaccessible planets. Virtual reality technology has changed people's new travel experience; subverted people's understanding of future travel, sightseeing, and tourism; and has become an important direction of cultural tourism development [14]. Compared with the innovation of 3D movies, IMAX movies, and other pictures, the changes in language and audiovisual technology of VR can be called innovative reinnovation. Traditional film is a two-dimensional image display, while VR film is 360 degrees, which can be used to construct a three-dimensional image space [15]. VR movies are very similar to movie games. The audience can choose different angles, fully immerse themselves in the story, and experience various progress and understanding of the progress of the story.

VR education is an abstract theoretical route and practical training. VR technology changes teaching methods; the construction of models and the display of molecular structures of compounds in a wide range of fields are provided to students in a virtual learning environment [16]. A virtual experience can be provided to improve the learning process of students. On the one hand, through the trilogy of human body structure, materials, abstract knowledge, and other aspects of structure, students can learn intuitively. On the other hand, in terms of chemical reactions, the 
virtualization of risk tests can improve experience [17] and continuously improve students and their skills. This may become a model of rich educational content. VR technology has penetrated into existing firmware models. Through virtual reality technology, students can learn theoretical knowledge, deepen their understanding, improve the learning environment, increase interaction, learn more interesting, and feel the special experience of stimulation. In order to improve the simulation-related natural environment and social environment, students can pass the time and space environment. The simulation of the interaction between them enables relevant experts to quickly acquire knowledge [18]. VR education content provides a platform for teachers, and educational virtual reality technology equipment as an additional education method has broad market potential. Virtual reality technology can create a good shopping environment, greatly improving and enriching customers' online shopping experience [19].

Edge computing refers to the use of an open platform that integrates network, computing, storage, and application core capabilities on the side close to the source of things or data to provide nearest-end services. Its applications are initiated on the edge side to generate faster network service response and meet the basic needs of the industry in realtime business, application intelligence, security, and privacy protection. Edge computing is between physical entities and industrial connections or at the top of physical entities. And cloud computing, you can still access the historical data of edge computing.

Multimedia edge computing mainly provides external services in the form of virtual machines. Therefore, the problem of resource allocation is the placement of virtual machines under the premise of meeting business needs. The use of efficient resource allocation mechanisms is an inevitable hot issue in the media cloud. It helps improve the level of multimedia file design and production, image rendering, composition, and other related fields; the difficulty of building a data center in cloud computing is relatively low; the scale of the data center can be dynamically adjusted; and cloud tasks can be between nodes. Fast feedback and efficient execution can realize the interactive design of multimedia conferences, the simulation and debugging of audio and video effects, and the rapid design and creation of media content, which greatly improves the overall ability of multimedia, film and television, and animation design and production.

\subsection{The Spreading Method of Taijiquan and Its Restrictive} Factors. Taijiquan culture is one of the important contents of Chinese traditional culture. It has absorbed and integrated the essence of many social fields in the process of long-term development and popularization and has a strong humanitarian color and national characteristics [20]. In the context of cultural diversity and economic globalization, Taijiquan culture, as one of the cultural representatives with oriental characteristics, has attracted more and more attention. At present, the research on the popularization of Taijiquan mainly focuses on the three fields: personal fitness, physical education, and technical theory [21]. The Internet has brought people into the information age. Online media has become the mainstream media in China. The new way of using online media means that the popularization of Taijiquan culture in China will have a major impact on the sustainable development of Chinese martial arts. China's Taijiquan culture is rich in content and diverse in forms. With the continuous development of global cultural diversity, the new martial arts gradually replaced the Chinese martial arts culture under the influence of the western sports concept [22]. In order to effectively protect our traditional Taijiquan culture, we must not only protect our fitness, science, market orientation, and competitiveness but also pay attention to mining, screening, protecting, and researching Taijiquan culture. After the information is collected and digitized, the overall situation of Taijiquan culture can be reproduced objectively, which is helpful for the communication and research of Taijiquan culture. Users can search and read literature related to Taijiquan at any time. The Internet is the latest high-tech technology. Tai Chi can process-related information. Therefore, various forms of Tai Chi culture in the cyberspace of regional culture, such as photos, sounds, texts, videos, and 3D models, can be reproduced objectively and comprehensively. The preservation, the inability to lose, or the damaged classical reproduction of Tai Chi can be restored [23]. An information platform can be provided for Taijiquan fans to acquire, understand, and exchange Taijiquan culture, and a rich information foundation can be provided for Taijiquan culture researchers $[24,25]$.

There are many limiting factors in the international spread of Tai Chi; the biggest one is the barrier of language communication. Therefore, the external communication problem of Taijiquan, in order to solve the bottleneck, is the correct and standardized translation text. The essence and style of this cultural treasure bought by China for thousands of years is a method to prove to the world [26]. At present, the number of translated documents related to Taijiquan is gradually increasing, but the level is uneven, and there are many contradictions in the translation of professional terms. The lack of unified norms and standards will not only cause confusion and misunderstandings for international fans who want to understand and learn Taijiquan but also have a negative impact on the international exchanges of the national cultural brand Taijiquan. Therefore, the standardization and unified translation of Taijiquan is the prerequisite and basis for Taijiquan to enter the world. When translating Taijiquanrelated content, we should not only pay attention to the translation of vocabulary and terms but also clearly explain the technical nature of Taijiquan and pay attention to the interpretation of its cultural connotation [27]. Relevant statistics show that the promoters of Tai Chi's international promotion and development are mainly Tai Chi fans, athletes, and overseas Chinese fans. These people are familiar with the skills, knowledge, and culture related to Tai Chi but still have serious shortcomings in cultural exchanges. At the same time, due to differences in language and education levels, the number of comprehensive 
abilities with precise language skills and Tai Chi knowledge and skills has further reduced, which further limits the progress of international exchanges in Tai Chi [28].

According to the status quo of the research and dissemination of Taijiquan, the problems that need to be solved urgently are the lack of unified and authoritative translationrelated terms and conditions in the process of international communication and development of Taijiquan, and the lack of internationally influential Taijiquan-related classics and references [29]. Books, foreign uneven quality versions of Tai $\mathrm{Chi}$, and audio, video, and application software related to the quality of Internet-based Tai Chi are relatively scarce [30]. As a representative of traditional Chinese culture, spreading Tai Chi's Eastern and Western cultural foundations requires good background knowledge and comprehensive ability to be competent in this difficult task: on the one hand, spreading requires a good understanding of the basic knowledge about Tai Chi, and on the other hand, they need to have a good foreign language proficiency and solid intercultural communication skills. However, due to many reasons, the number of talents with the above two aspects are relatively small, leading to the current international communicators of Taijiquan culture being mostly Taijiquan coaches who master the routines of Taijiquan and lack the ability of cross-cultural communication [31]. The content of its dissemination is also limited to the boxing skills and movements of Taijiquan, and the promotion and dissemination of Taijiquan culture are very limited. Therefore, how to cultivate the promoters of Tai Chi culture in the new era is one of the problems that need to be solved. If organizations or individuals without professional knowledge and abilities are allowed to take the lead in the promotion of Tai Chi, it will have a negative impact on my country's Tai Chi culture and national image.

\section{Related Experiments of VR Technology Application and Taijiquan Teaching}

3.1. Purpose. Chinese traditional Tai Chi is undoubtedly the most representative type of boxing. Taijiquan is a comprehensive boxing technique that combines fitness and martial arts. Its movement characteristics are dependent on movement and static, speed, and slow, and it has significant curative effects on the treatment of various chronic diseases. It is highly respected worldwide. It is reported that there are more than 300 million Tai Chi fans in the world, and tens of thousands of people participated in a Tai Chi cultural exchange conference in South Korea. Authoritative media and research institutions in many countries have listed Tai Chi as one of the top ten most suitable human fitness programs.

The purpose of this experiment is to explore the role of VR technology in the spread and promotion of Taijiquan. To achieve the promotion of Taijiquan, it must first be inherited. The inheritance and spread of Taijiquan cannot be separated from learners, so improvement in the sensory enjoyment of Tai Chi learners and the mobilization of students' enthusiasm for spontaneous learning are very important for the promotion and dissemination of Tai Chi. This article uses VR technology to develop new Taijiquan teaching to understand whether VR technology has a certain impact on students' learning enthusiasm compared with ordinary slide courseware, teacher handwriting on blackboard, observation video, and other teaching methods in Taijiquan teaching. Technology enters Taijiquan class to provide theoretical support.

3.2. Subjects and Groups. In this experiment, 40 students in the 24-style Taijiquan elective course were taken as the research objects. The elective students were randomly divided into an experimental group and a control group, with 20 students in both groups. A comparison design between groups was used to test the difference between the two groups. The experimental group used VR technology courseware and the subjects' technical videos before and after teaching for comparative analysis. The control group used traditional teaching and training methods. The teaching progress and teaching time of the two groups are equal. With unified class hours, the same teaching materials about Taijiquan, and the same teacher, two groups of students learn Taijiquan separately, two classes per week ( 3 class hours, 50 minutes, all technical classes), a total of 24 class hours.

3.3. Design of Tai Chi VR Courseware. Based on VR technology, this paper improves the teaching method of Taijiquan, uses 3ds Max VR design software to design the VR courseware of 24-style Taijiquan and applies VR technology to the teaching of Taijiquan. Courseware design: Taijiquan VR courseware mainly includes Taijiquan exercise introduction, practice points, practice methods and techniques, action diagrams, a full set of action demonstrations, segmented action presentations, and analysis of common wrong actions. System: 3ds Max VR software is used for courseware production, and superjieba2010 is used for video editing. The function of the courseware is an interactive interface that is simple to operate, easy to learn, and easy to use. The courseware is compiled into the Windows operating system and runs independently. The basic environment of courseware application: Pentium or above microcomputer, Windows 98 or above Chinese version operating system, vga00 $\times 600$ monitor, sound card, and CD-ROM drive $(1 \mathrm{~g}$ hard disk or above). The main function of VR courseware: it can realize the function of automatic demonstration. Each part has action diagrams and movie files, which can be demonstrated and explained automatically. Interactivity: each part can be converted by clicking the corresponding button. Controllability: the corresponding button is used to control the switching of the system sound, which can be paused and started at any time and can be combined with instructions and played repeatedly.

3.4. Tai Chi Learning Process. Combining the teaching content of Taijiquan, the appropriate VR software and VR scenes was chosen, and the VR teaching resources that Taijiquan courses need to use was constructed. After understanding 3D broadcasting, Blu-ray VR, tutor, veer, 
orange, and other VR software and comparing the number, content, definition, and functions of the video library, I chose tutor and $3 \mathrm{D}$ broadcasting as the applications used in the experiment. Before the Taijiquan course officially started, the students in the experimental group were trained in the operation method of VR equipment, instructed the students to use VR software, and adjusted the pupil distance and object distance of the VR head display until the image was clear, so as not to affect the learning effect due to the unsmooth use of equipment in the classroom. The Tai Chi class in this experiment is mainly conducted by students. Students in the experimental group were asked to use virtual reality equipment to assist in the construction of Tai Chi situations and then discussed to exchange experiences. Taijiquan coaches will make supplementary comments and explain and summarize the content of knowledge during the students' discussion so as to enhance students' understanding of knowledge. In the teaching process of the control group, no VR equipment was used, and traditional teaching methods such as PPT slides and $2 \mathrm{D}$ video playback were still used. Data were collected from Tai Chi students, lecturers, and researchers. In response to the problems in the research, combined with the specific situation of the Taijiquan course, the outline of student interviews was designed, and the students of each experimental group were interviewed to understand the students' sentimental attitudes and attitudes towards Taijiquan learning. Process evaluation: Taijiquan coaches of four majors used the Chinese Taijiquan standard as the scoring standard, assessed the learning status of the two groups of students, and tested the students' understanding of Taijiquan and their mastery of skills.

3.5. Index Scoring by Questionnaire Survey. Virtual reality technology has been used in the process of Tai Chi course design. Tai Chi teaching training uses VR technology. Learners can play a role in the virtual space and practice a skill wholeheartedly. The practitioner can repeat the feedback given by the system and practice until they master this skill. VR technology will provide a safe training environment so that practitioners' Tai Chi skills and technical training have a virtual space where they can directly interact.

According to the requirements of this research, three questionnaires have been developed on the basis of a large number of materials related to this article. In order to understand the basic situation of students learning Taijiquan and the possibility of Taijiquan being spread and promoted, questionnaire 1 "Questionnaire survey on whether you are interested in VR technology combined with Taijiquan" was designed. In order to ensure the implementation of the experiment, questionnaire 2 was carefully designed "A questionnaire survey of whether you want to try Taijiquan learning based on VR technology," and questionnaire 3 "A questionnaire survey of whether VR panoramic video is beneficial to the spread and promotion of Taijiquan in China" was also designed. After the teaching test, all students have been tested theoretically and professionally tested the test papers. By adopting the repeated test method, the reliability of the questionnaire was effectively tested, and the same questionnaire was measured repeatedly at different times, with an interval of 10 days as a measurement cycle. The correlation analysis of the two measurement results or the difference test was used to evaluate the reliability of the questionnaire. We also conducted reliability tests on 10 related experts. The correlation coefficient of the research questionnaire is 0.93 , which proves the credibility and importance of the research results. The statistical data of the recovery rate of the questionnaire survey are shown in Table 1.

\section{Experimental Results of Taijiquan's Promotion in VR Technology}

4.1. Analysis of the Experimental Results of VR Multimedia Technology Applied to Taijiquan Teaching. Through the experimental research on the optional teaching of 24-style Taijiquan, the teaching effect of VR multimedia teaching technology in technical courses is tested. The teacher in the teaching and research section does not know the students in the experimental group and the control group. Randomly disrupt the order of technical assessment of students in the experimental group and the control group. After the Taijiquan class is over, 4 Taijiquan coaches will simultaneously evaluate the skills of the experimental group and the control group. The study found that in the teaching test, the experimental group had an $18 \%$ higher rate of excellence, a $15 \%$ higher rate of goodness, a $22 \%$ reduction in the number of middle-level students, and a $14 \%$ reduction in the number of passing people. The proficiency of basic techniques of Taijiquan in the experimental group was significantly higher than that in the control group. The technical assessment scores of the two groups of students are shown in Table 2.

The results of the study show that the Taijiquan coaches' scores for the experimental group's skill evaluation are higher than those of the control group. After an independent sample $T$ test with SPSS15.0 software, it can be concluded that the test results of the experimental group and the control group are $P<0.01$, which is very significantly different. From the comparison result, the students in the experimental group have a significantly higher level of mastery of Tai Chi techniques than the students in the control group. In the 24-style Taijiquan technique teaching, using VR technology and multimedia-assisted teaching methods, the teaching effect of the experimental group of students' technical evaluation index was significantly higher than that of the control group with traditional teaching methods. During the same period of time, the experimental group using VR multimedia technology was significantly more proficient in Taijiquan than the control group, indicating that VR multimedia technology is beneficial to improving the learning efficiency of Taijiquan. The research results show that the application of VR technology to the multimedia technology of Taijiquan can improve the learning efficiency of Taijiquan. The relevant data is shown in Figure 1.

From the data in Figure 1, it can be seen that the application of VR technology to Taijiquan multimedia technology can improve the learning efficiency of Taijiquan, and 
TABLE 1: Statistics on the recovery rate of the questionnaire.

\begin{tabular}{lccc}
\hline Questionnaire type & Issue questionnaire & Effective response to questionnaires & Effective recovery rate (\%) \\
\hline Questionnaire 1 & 200 servings & 192 servings & 96 \\
Questionnaire 2 & 100 servings & 100 servings & 100 \\
Questionnaire 3 & 100 servings & 100 servings & 100 \\
\hline
\end{tabular}

TABLE 2: Technical assessment scores of two groups of students.

\begin{tabular}{|c|c|c|c|c|}
\hline Group & Excellent & Good & Medium & Pass \\
\hline Test group & 11 & 5 & 3 & 1 \\
\hline Test group & 8 & 5 & 5 & 2 \\
\hline Ratio of two groups (\%) & 128 & 100 & 60 & 50 \\
\hline
\end{tabular}

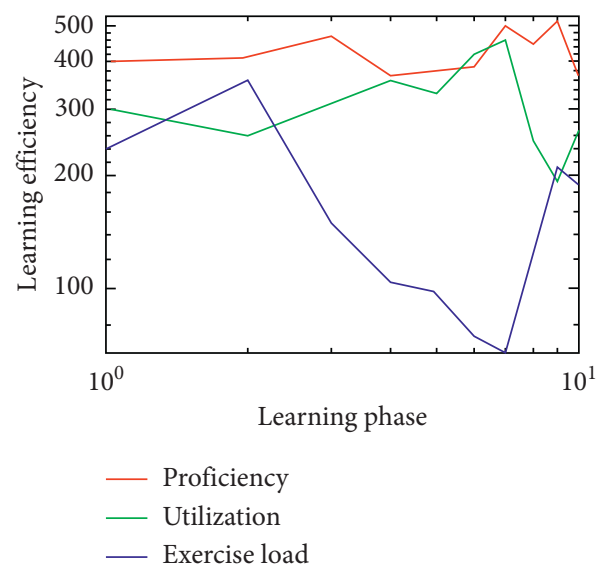

FIgURE 1: The effect of VR technology applied to Taijiquan multimedia technology on the efficiency of Taijiquan learning.

the overall learning efficiency of Taijiquan has increased by $34.8 \%$. The design of comparison between groups was used to test the difference between the two groups. The experimental group used CAI courseware and the subjects' technical videos before and after teaching for comparative analysis; the control group used traditional teacher teaching and training methods for teaching. The teaching progress and teaching time of the two groups are equal. With unified class hours, the same textbooks, and the same teacher, two groups of students will learn Taijiquan separately, once a week.

The results of the questionnaire survey showed that the traditional teaching methods of Taijiquan were not only tedious but also brought great difficulties to teachers. Therefore, students are easy to withdraw when encountering difficulties and will not stimulate their interest in learning. In the Taijiquan teaching of VR multimedia system, the image, text, music, animation, and other information are vividly, concretely, and intuitively displayed in front of the students, which refreshes the students and greatly improves their visual and auditory experience. Students can intuitively feel the characteristics of Tai Chi, understand the rich connotation of Tai Chi, and inject new vitality into the dull learning atmosphere. At the same time, due to the unique interactivity of VR multimedia, students can carefully observe the demonstration actions from different angles, speeds, and different directions so that the impression formed by the brain is clearer and more specific so that students can understand the expression. Repeated movements have fundamentally changed students' perceptions of Tai Chi and stimulated their interest and enthusiasm for learning. The research results show that the application of VR multimedia technology to Taijiquan teaching is beneficial to improve the visual and auditory effects of Taijiquan learners, thereby increasing students' enthusiasm for learning Taijiquan. The specific data are shown in Figure 2.

It can be seen from the data in Figure 2 that the application of VR multimedia technology to Taijiquan teaching is conducive to improving the visual and auditory effects of Taijiquan learners, thereby improving students' enthusiasm for learning Taijiquan and enhancing the visual and auditory effects of students in the learning process. Students' learning enthusiasm increased by $25.3 \%$ overall.

4.2. Analysis of the Effect of VR Multimedia Technology on Taijiquan Promotion. VR is the English abbreviation of virtual reality technology. It is a new high-end man-machine interface information technology that has just been developed at the end of the 20th century and is also the fastest growing in the past decade. The VR system uses image processing technology, multimedia technology, computer graphics, artificial intelligence, and Internet technology to naturally integrate various abstract information. And use a 


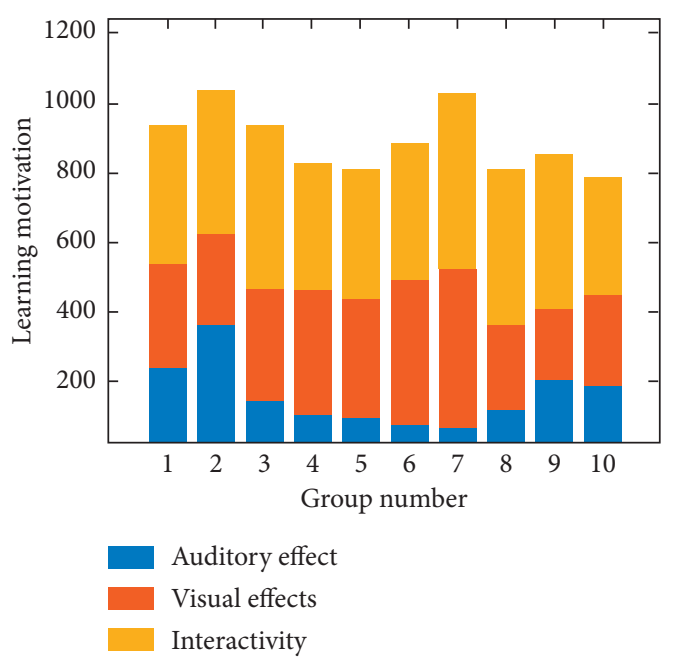

Figure 2: The impact of VR multimedia technology in Tai Chi teaching on the visual and auditory effects of Tai Chi learners and their motivation to learn.

variety of sensing devices (such as $3 \mathrm{~d}$ helmets, clothing, data gloves, and $3 \mathrm{~d}$ mouse) for simulation and real-time interaction, simulating a way to allow users to create a real-time three-dimensional image world reflecting the changes and interactions of entities, etc. Make people have a more real feeling and understanding of the computer virtual world, promote human-computer interaction, and let the experiencer have a sense of immersion, imagination, and interaction.

The results of the questionnaire survey show that the most important way to spread Tai Chi is through multimedia, the Internet, and friends introducing it to each other. The Tai Chi courses offered by the school are mainly through movies, TV series, and media reports. The most important way for Tai Chi enthusiasts to learn Tai Chi is to learn from friends and schools around them. Some people learn Tai Chi through introduction and dissemination. The school also offers Tai Chi courses. Therefore, the promotion and dissemination of Tai Chi mainly focus on traditional one-toone teaching. The experimental results show that the use of virtual reality technology in teaching can improve students' sports performance, and the results are quite different from traditional teaching methods. The application of VR technology stimulates students' enthusiasm for learning Taijiquan, makes full use of students' senses, especially vision; greatly enhances the leading role of teachers in the classroom; and reduces teachers' subjectivity. Virtual reality technology teaching can show the wrong movements of Tai Chi and the correct technical movements at the same time so that students can see, think, and compare themselves so as to improve and prevent students' wrong behaviors. While learning Taijiquan skills, students have cultivated their comprehensive abilities in observation, analysis, and problem-solving. The application of VR multimedia technology arouses students' interest in learning, increase students' positive thinking, avoids indoctrination, and develops students' creative thinking. The application of VR multimedia technology increases the attractiveness of Taijiquan and is beneficial to the spread and promotion of Taijiquan. The research results show that the use of VR multimedia technology of Taijiquan has improved the attractiveness of Taijiquan. The relevant data are shown in Figure 3.

From the data in Figure 3, it can be seen that the use of VR multimedia technology in Taijiquan has increased the attractiveness of Taijiquan. The questionnaire results show that the attractiveness index of Taijiquan has increased by $36.3 \%$, which has attracted more and more people to join.

The survey found that VR technology has been applied in the process of Tai Chi course design. Using VR technology in Tai Chi teaching and training, learners can play roles in virtual spaces and practice their skills wholeheartedly. Practitioners can practice repeatedly according to the feedback of the system until they master the skill. VR technology will provide a safe training environment so that practitioners' Tai Chi skills and technical training have a virtual space where they can directly interact. The application of VR technology in Taijiquan teaching mainly includes the following aspects. First of all, according to this kind of VR technology, teaching courseware of Tai Chi movements can be made. In this way, students can overcome the following shortcomings: in video teaching, they can only observe a certain technical action from one angle but cannot accurately see and appreciate the action in actual teacher demonstrations. Secondly, the development of online teaching resources for Taijiquan and the use of the Internet to implement remote teaching have avoided the registration fees and cumbersome learning steps of Taijiquan training classes and greatly reduced the cost of learning. Thirdly, in the virtual reality environment, students can confidently and boldly fight against virtual opponents without worrying about any danger, thus increasing the spread of Tai Chi. The research results show that VR multimedia technology can reduce the cost and danger of learning Taijiquan. The specific data are shown in Figure 4. In traditional physical education, teachers can only guide students' learning by 


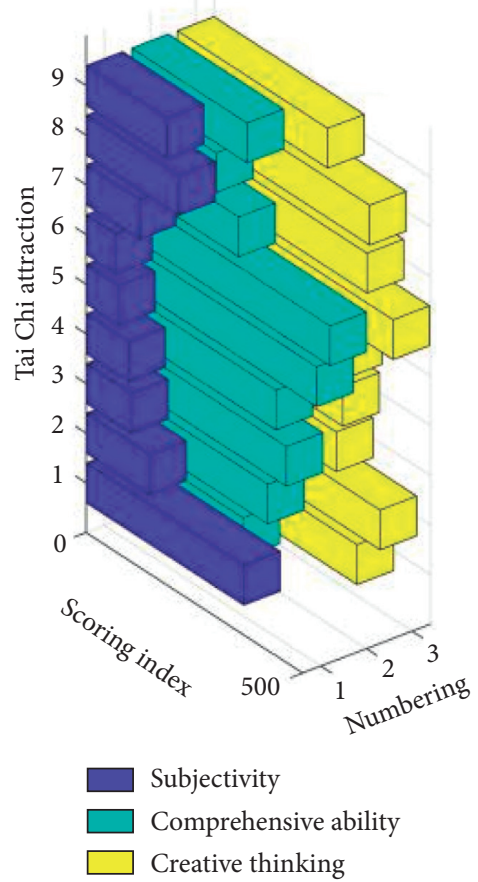

FIgURE 3: The use of VR multimedia technology of Taijiquan can improve the attractiveness of Taijiquan.

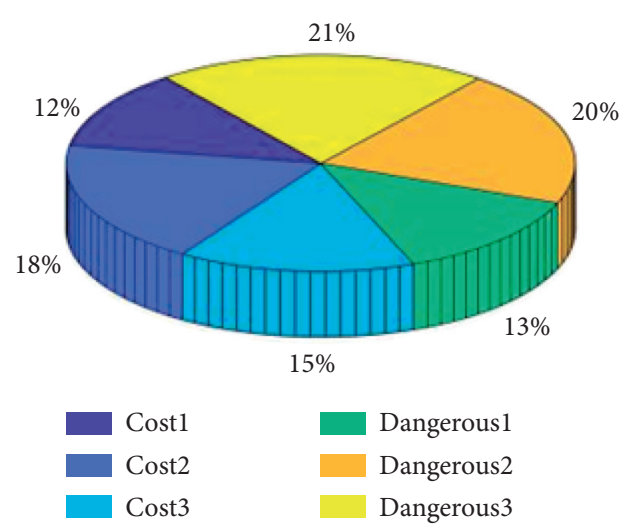

FIGURE 4: The effect of VR multimedia technology on the cost of learning Tai Chi and the risk index in the learning process.

relying on their own teaching experience, teaching content, schedule, class hours, and assessment standards. Due to the differences of students in intelligence, physical fitness, physical education, and so on, there are differences in students' understanding, mastery, and learning of each technical action and the wrong technical actions produced in the learning process are also different. At this time, Tai Chi is very dangerous.

It can be seen from Figure 4 that VR multimedia technology can reduce the cost and danger of learning Taijiquan. Compared with registering for training classes, the cost of learning Taijiquan is reduced by $74.5 \%$, and the risk of learning Taijiquan is reduced by $56.9 \%$ so that more people have the opportunity and courage to learn Taijiquan, greatly improving the spread and promotion of Taijiquan.

\section{Conclusions}

(1) Taijiquan is a representative sport in my country's traditional sports. It occupies an important position in China's traditional sports. It has been rated as my country's intangible cultural heritage. Widespread and promotion of Taijiquan can promote my country's traditional culture. VR technology is an augmented reality technology based on sensors and computers. Combining VR technology with the advantages of Taijiquan is beneficial to change the traditional teaching methods and the spread of Taijiquan.

(2) The research results show that the combination of VR technology and Tai Chi teaching can increase the visual and auditory effects of students in the learning process by $28.4 \%$. It is really uncomfortable to give students an immersive experience, and students' learning enthusiasm increases by $25.3 \%$. The overall learning efficiency has increased by $34.8 \%$, and people's interest in learning Tai Chi has also increased. Therefore, it can be seen that the application of VR multimedia technology to Tai Chi teaching is feasible.

(3) The study found that the use of VR multimedia technology in Taijiquan has increased the attractiveness of Taijiquan. The questionnaire results showed that the attractiveness index of Taijiquan has increased by $36.3 \%$, which has attracted more and more people to participate in the study of Taijiquan. VR multimedia technology can reduce the cost and danger of learning Tai Chi. Compared with registering for training classes, the cost of learning Tai Chi 
is reduced by $74.5 \%$. At the same time, the risk of learning Tai Chi is reduced by $56.9 \%$, enabling more people with the opportunity and courage to learn Tai Chi; the spread and promotion of Tai Chi has been greatly improved.

\section{Data Availability}

This article does not cover data research. No data were used to support this study.

\section{Conflicts of Interest}

The authors declare that they have no conflicts of interest.

\section{References}

[1] Y. Kobayashi, "Preface to the special issue on "challenge and progress of process technology and fundamental research for the promotion of lime dissolution into slag", " ISIJ International, vol. 57, no. 10, p. 1669, 2017.

[2] L. Fa-Mei, S. Shao-Ping, and H. E. Zhi-Gang, "Research on the method of comparing the in-situ performance of the domestically made XBT and analysis of the results," The Journal of Ocean Technology, vol. 17, no. 11, pp. 16-19, 2019.

[3] X. Zhou, Z. Zhong, X. Bian et al., "Indoor wideband channel measurements and analysis at 11 and $14 \mathrm{GHz}$," IET Microwaves, Antennas \& Propagation, vol. 11, no. 10, pp. 1393-1400, 2017.

[4] C. T. Chuang, "Criterions of normality of families of meromorphic functions and applications," Radiotherapy \& Oncology, vol. 78, no. 3, pp. 332-338, 2015.

[5] H. Cheng, N. Yu, and T. J. Wang, "Non-supervised learning for spread spectrum signal pseudo-noise sequence acquisition," Journal of Electronic Science and Technology, vol. 13, no. 1, pp. 83-86, 2015.

[6] H. Kurokochi and K. Toyama, "Invasive tree species Robinia pseudoacacia: a potential biomass resource in Nagano prefecture, Japan," Small-Scale Forestry, vol. 14, no. 2, pp. 205-215, 2015.

[7] Y. Ohara, T. Enomoto, A. Hara et al., "A case of anal canal adenocarcinoma with perianal pagetoid spread treated by laparoscopic abdominoperineal resection," Nihon Gekakei Rengo Gakkaishi (Journal of Japanese College of Surgeons), vol. 41, no. 5, pp. 803-807, 2016.

[8] C. Zhang, C. Chen, and W. Shen, "Impact of population movement on the spread of 2019-nCoV in China," Emerging Microbes and Infections, vol. 53, no. 14, pp. 19-28, 2020.

[9] J. M. Ko, H. J. Park, and C. H. Kim, "Clinicoradiologic evidence of pulmonary lymphatic spread in adult patients with tuberculosis," American Journal of Roentgenology, vol. 204, no. 1, pp. 38-43, 2015.

[10] D. P. Gaillot, E. Tanghe, W. Joseph et al., "Polarization properties of specular and dense multipath components in a large industrial hall," IEEE Transactions on Antennas and Propagation, vol. 63, no. 7, pp. 3219-3228, 2015.

[11] T. Schweiger, K. Hoetzenecker, S. Taghavi, and W. Klepetko, "Extended cervico-thoracic metastasectomy for testicular non-seminomatous germ cell tumour masses through an inverse T and combined collar incision," European Journal of Cardio-Thoracic Surgery, vol. 47, no. 5, pp. 931-933, 2015.

[12] D. M. Rayan and M. Linkie, "Conserving tigers in Malaysia: a science-driven approach for eliciting conservation policy change," Biological Conservation, vol. 184, no. 36, pp. 24-26, 2015.

[13] D. Zhou, "The development status of VR technology and its application field research practice," Electronic Technology and Software Engineering, vol. 25, no. 17, pp. 147-148, 2018.

[14] H.-C. Lin, C.-H. Wang, F.-J. Tsai et al., "Enterovirus infection is associated with an increased risk of childhood type $1 \mathrm{di}-$ abetes in Taiwan: a nationwide population-based cohort study," Diabetologia, vol. 58, no. 1, pp. 79-86, 2015.

[15] X. Ren, Y. Che, K. Yang, and Y. Tao, "Risk perception and public acceptance toward a highly protested waste-to-energy facility," Waste Management, vol. 48, no. 6, pp. 528-539, 2016.

[16] O. S. Al-Humaidan, T. A. El-Kersh, and R. A. Al-Akeel, "Risk factors of nasal carriage of Staphylococcus aureus and methicillin-resistant Staphylococcus aureus among health care staff in a teaching hospital in central Saudi Arabia," Saudi Medical Journal, vol. 36, no. 9, pp. 1084-1090, 2015.

[17] J. D. Scasta, E. T. Thacker, and T. J. Hovick, "Patch-burn grazing (PBG) as a livestock management alternative for fireprone ecosystems of North America," Renewable Agriculture and Food Systems, vol. 31, no. 06, pp. 550-567, 2016.

[18] F. Mingozzi and F. Boisgerault, "The skeletal muscle environment and its role in immunity and tolerance to AAV vector-mediated gene transfer," Current Gene Therapy, vol. 15, no. 4, pp. 150-153, 2015.

[19] S. Mehrabian, J. Extra, Y. H. Wu, M Pino, L Traykov, and A. S Rigaud, "The perceptions of cognitively impaired patients and their caregivers of a home telecare system," Medical devices (Auckland, N.Z.), vol. 8, no. 17, pp. 21-29, 2015.

[20] W. Duan, Z. Fan, P. Zhang, G. Guo, and X. Qiu, "Mathematical and computational approaches to epidemic modeling: a comprehensive review," Frontiers of Computer Science, vol. 9, no. 5, pp. 806-826, 2015.

[21] Z. Wanfang, D. Shaojun, and G. Huifang, "The chitinase C gene PsChiC from Pseudomonas sp. and its synergistic effects on larvicidal activity," Genetics and Molecular Biology, vol. 38, no. 3, pp. 366-372, 2015.

[22] C. Holzmann, D. N. Markowski, I. VON Leffern, T Löning, and J Bullerdiek, "Patterns of chromosomal abnormalities that can improve diagnosis of uterine smooth muscle tumors," Anticancer Research, vol. 35, no. 12, pp. 6445-6456, 2015.

[23] Yu Yang, "Research on restrictive factors and countermeasures of Taijiquan international spread and development," Journal of Mudanjiang Education Institute, vol. 195, no. 12, pp. 72-74, 2018.

[24] Z. Lv, X. Li, and W. Li, "Virtual reality geographical interactive scene semantics research for immersive geography learning," Neurocomputing, vol. 254, pp. 71-78, 2017.

[25] Z. Lv, D. Chen, R. Lou, and Q. Wang, "Intelligent edge computing based on machine learning for smart city," Future Generation Computer Systems, vol. 115, pp. 90-99, 2021.

[26] F. T. Guo, Z. W. Su, and G. Y. Wang, "Prediction model of human-caused fire occurrence in the boreal forest of Northern China. Ying yong sheng tai xue bao = the journal of applied ecology/Zhongguo sheng tai xue xue hui," Zhongguo ke xue yuan Shenyang ying yong sheng tai yan jiu suo zhu ban, vol. 26, no. 7, pp. 209-211, 2015.

[27] H. Chih-Weim, L. Chang-Hsien, and F. Hsiu-Lung, "Clinical features and computed tomography characteristics of nonKlebsiella pneumoniae liver abscesses in elderly ( $>65$ Years) and nonelderly patients," Yonsei Medical Journal, vol. 56, no. 2, pp. 519-528, 2015. 
[28] L.-Y. Yeh, P.-Y. Chiang, Y.-L. Tsai, and J.-L. Huang, “Cloudbased fine-grained health information access control framework for lightweight IoT devices with dynamic auditing and attribute revocation," IEEE Transactions on Cloud Computing, vol. 6, no. 2, pp. 532-544, 2018.

[29] S. Wan, L. Qi, X. Xu, C. Tong, and Z. Gu, "Deep learning models for real-time human activity recognition with smartphones," Mobile Networks and Applications, vol. 25, no. 2, pp. 1-13, 2019.

[30] R. A. B. Badawy, S. J. Vogrin, A. Lai, and M. J. Cook, "Does the region of epileptogenicity influence the pattern of change in cortical excitability?" Clinical Neurophysiology, vol. 126, no. 2, pp. 249-256, 2015.

[31] Y. Zhang, H. Pang, and L. Zhou, "Research on the promotion of taijiquan in multimedia technology_taking VR technology as an example," Wushu Research, vol. 3, no. 5, pp. 66-68, 2018. 